\title{
ELECTROCHEMICAL REDUCTION OF 4-AMINOPYRIMIDINE AND CYTOSINE IN DIMETHYL SULFOXIDE
}

\author{
TAMOTSU WASA * and PHILIP J. ELVING \\ The Unioessity of Mictigan, Ann Arbor. MI 48109 (U.S.A.)
}

(Received 29th March 1982; in revised form Ist June 1982)

\begin{abstract}
The electrochemical reduction in dimethyl sulfoxide of cytosine (4-amino-2-hydroxypyrimidine) and one of its model compounds (4-aminopyrimidine) has been examined. Initially. each pyrimidine (FiH) undergoes a reversible diffusion-controlled one-electron reduction of the $3,4 \mathrm{~N}=\mathrm{C}$ double bond to the radical anion $\left(\mathrm{RH}^{-}\right)$, which can dimerize or can react with the parent compound (father-son reaction) to form the neutral free radical $\left(\dot{R}_{2}\right)$ and the pyrimidine anion $\left(\mathrm{R}^{-}\right)$; the radical can dimerize or be further reduced perhaps after effective protanation: the anion forms a redox couple with $\mathrm{Hg}(\mathrm{I})-\mathrm{Hg}(0)$. Other coupled reactions, which may occur under suitable conditions, include reaction between anionic dimer ( $-\mathrm{RH}-\mathrm{RH}^{-}$) and $\mathrm{RH}$, proton-assisted decomposition of dimer to form reducible $\dot{\mathrm{RH}}_{3}$. and deamination of the two-electron reduction product $\left(\mathrm{RH}_{3}\right)$. which is a gem diamine to generate 2-hydroxypyrimidine or pyrimidine itself. The effects on the electrochemical redox patiem of added uater. strong acid $\left(\mathrm{HCOO}_{4}\right.$ ). weak acid (chloroacetic and benzoic acids), and strong base (Et $4 \mathrm{NOH}$ ) are described.
\end{abstract}

\section{INTRODUCTION}

In spite of the several papers published on the polarography of cytosine (4-amino2-hydroxypyrimidine) in aqueous media [1-7], there still seems to be some question concerning its reduction mechanism [8]- Since cytosine is one of the two nitrogen heterocyclic bases normally electrochemically reducible in nucleic acid (DNA and RNA) fragments (adenine is the other), its reduction mechanism is of considerable interest, for example, to those using electrochemical techniques to monitor investigations of the nucleic acids.

In order to clarify the situation and to provide additional desired information. the polarographic behavior in aqueous and nonaqueous media of 4-aminopyrimidine (4-AP), 2-hydroxypyrimidine (2-HP). cytosine and related compounds (Fig. 1) is being investigated with particular attention to the roles of free radicais and anionic species (radical anions, carbanions, and dissociated conjugate bases) as reaction intermediates. In addition to being a model compound for cytosine. $4 A P$ is also the logical bridge for comparing the two major nucleic acid bases of cytosine and aderine (6-aminopurine), e.g., in respect to influence of the amino group on

- Permanent address: Department of Applied Chemistry, University of Osaka Prefecture. Sukzi. Japan. 
<smiles>C=C=C1N=CC=CNC1=O</smiles>
2-HP

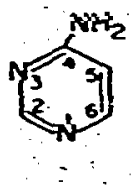

4-AP<smiles>Nc1ccnc(O)n1</smiles>

Cy:

Fig I. Formula for 2-hydraxypyrimidine (2-HP), 4-aminopyrimidine (4-AP), and cytosine (4-amino-2hydroxspyrimidines Cy) In both aqueous and nonaqueous media; 2-hydroxypyrimidine and cytosine exist Largely. if not entirely, in the keto form.

reduction ease and path; the pyrimidine 4-position and the purine 6-position are equivalent.

The present paper reports the results obtained for 4-AP and cytosine in nonaqueous media (dimethyl sulfoxide, DMSO), including the effects of added water, strong and weak acids, and strong base.

The electrochemical behavior of pyrimidines in aqueous media is summarized [9-11]; that of 4-AP has been recently examined [12] as has that of 2-HP and related comporomois (uracií; uridine; thymine) in nonaqueous media (DMSO) [13-16].

\section{Father-son reactions}

The term, father-son reaction, designates the situation where the principal product of a reaction reacts with the original primary reactant, e.g.,

$\mathbf{R}+$ reagent $=\mathbf{R}^{\circ}$

$\mathbf{R}^{\prime}+\mathbf{R}=$ products

where the reagent may be an electron [17].

Reactions of this general type have been encountered-or, at least, postulated-in connection with the electrochemical behavior of inorganic and organic species, e.g., a hydroxypyrimidine symbolized as $\mathrm{RH}$, can serve in nonaqueous media as a proton sourse for neutralization of the radical anion. which it forms on 1 e reduction, i.e.,

$\mathrm{RH}+e=\overline{\mathrm{RH}}^{-}$

$\overline{\mathbf{R}} \mathbf{H}^{-}+\mathbf{R H}=\overline{\mathbf{R}} \mathbf{H}_{2}+\mathbf{R}^{-}$

Such acid-base father-son reaclions also occur in studies published by Iversen, Baizer, Saveant and their co-workers.

Father-son reactions may cause observed polarographic wave heights to be less than expected for a 1 e process and coulometric $n$ values to be considerably less than one. 


\section{EXPERHAENTAL}

Chemicals

Cytosine (Nutritional Biochemical; Calbiochem) was used without further purification. 4-Aminopyrimidine (4-AP), prepared from 2-thiocytosine (Schwarz/Mann) by a modified Brown's method [18]. was twice vacuum sublimed; its purity was verified by m.p. $\left(151-152^{\circ} \mathrm{C}\right.$ [18], elemental analysis and NMR spectrum.

Dimethyl sulfoxide (Fisher Scientific), after having been dried over Linde 5A molecular sieves, was purified by on-line or off-line vacuum distillation. Tetraethylammonium perchlorate (TEAP), prepared following Kolthoff and Coetzee [19], was recrystallized four times from water and then dried in a vacuum oven at $60^{\circ} \mathrm{C}$. The background electrolyte system (0.1 $M$ TEAP in DMSO) gave residual currents of less than -0.1 and $0.3 \mu \mathrm{A}$ at +0.1 and $-2.7 \mathrm{~V}$, respectively. An occasional small wave $\left(E_{1 / 2}=c a-2.1 \mathrm{~V}\right)$ seemed to be due to a trace of $\mathrm{Na}(\mathrm{I})$; correction for this wave was readily made.

Other chemicals used were analytical reagent grade or the equivalent.

\section{Apparatus}

The electrochemical apparatus, including cells and electrodes used. have been described [13]. A mercury-plated platinum disk electrode (MPPDE; area $=0.50$ $\mathrm{mm}^{2}$ ) was used as indicator electrode for cyclic voltammetry of 4AP at very negative potential.

\section{Proceaures}

Vacuum line techniques [20] were used for test solution preparation until it was confirmed that the presence of less than $1 \%$ water and oxygen removal by nitrogen bubbling did not significantly affect the results. The test solution was then prepared by dissolving weighed amounts of compound and TEAP in freshly distilled DMSO and diluting to known volume. The effects of water, strong and weak acids, and strong base were examined by adding the following with a pipet: 10\% water in DMSO or pure water, $5.8 \times 10^{-2} M$ perchloric acid in DMSO (containing 0.75 .9 water); $5 \times 10^{-2} M$ benzoic or chloraacetic acid in DMSO; $6.8 \times 10^{-2} M$ tetraethylammonium hydroxide $\left(\mathrm{Et}_{4} \mathrm{NCH}\right.$ ) in DMSO (containing $9 \%$ water). After reagent addition, dissolved oxygen was removed by bubbling with dried nitrogen: measurements were made with nitrogen passing over the solution. Correction was made for dilution due to reagent solution addition.

\section{RESURTS AND DISCUSSION}

In both aqueous and DMSO media, a hydroxy substituent on a pyrimidine carbon, which is part of a $\mathbf{C}=\mathbf{N}$ bond, is largely removed as a result of a keto-enol 
equilibrium [21-24], i.e. cytosine and 2-HP are predominanty in the keto form

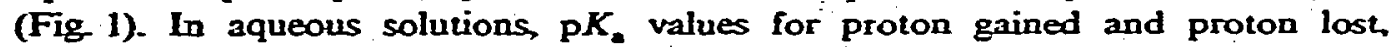
respectively, are 5.7 and 9.1 for $4 \mathrm{AP}, 4.5$ and 9.1 for cytosine and 2.2 and 9.2 for

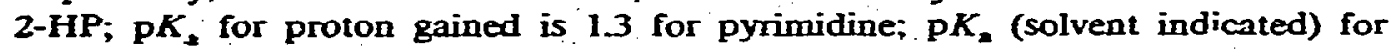
benzoic acid is $42\left(\mathrm{H}_{2} \mathrm{O}\right), 12.0$ (acetonitrile; $\mathrm{AN}$ ), 10.0 (DMSO) and 10.9 (N,N-dimethylformamide; DMF); for chloroacetic acid; it is $29\left(\mathrm{H}_{2} \mathrm{O}\right)$ and 9.8 (AN). Perchloric acid is essentially completely dissociated in all four solvents: TEAP is nearly completely dissociated in $\mathrm{H}_{2} \mathrm{O}$ and DMSO.

The DME polarographic and cyclic voltammetric behavior in DMSO of 4AP and cytosine are summarized in Table 1 and Fig. 2 Measurement of 4-AP voltammetric patterns is difficult owing to the closeness of the main cathodic wave to background discharge. Polarographic-waves and cyclic voltammetric peaks are designated by Roman numbers; suffixes a and $c$ indicate their anodic or cathodic nature; I designates the wave or peak due to the specific pyrimidine RH itself, II that due to protonated $\mathrm{RH}$, III due to the RH adduct with weak acid, IV due to mercury redox in presence of $\mathrm{R}^{-}$or $\mathrm{OH}^{-}$anion [the latter may be specified as $\mathrm{IVa}(\mathrm{OH})$ ], $\mathrm{V}$ due to mercury redox in presence of weak acid anion. VI due to hydrogen ion reduction. VII due to mercury redox in presence of adsorbed $\mathrm{OH}^{-}$, and VIII due to reduction of adsorbed protonated $\mathrm{RH}$ and protonated radical (cf. Fig. 13).

The primary electrode process is mainly diffusion controlled, e.g. $\log i-\log h$ plot slopes for wave Ic are near 0.5 and current functions ( $i_{\mathrm{p}} / A C D^{i} \mathcal{L}$ ) for peak Ic are constant with variation in cyclic voltammetric scan rate $(\mathrm{d} E / \mathrm{d} t=v)$.

The diffusion esrremt constami $\left(Y_{\mathrm{d}}\right)$ anư corrent iunction values for 4-AP-after

TABLE I

Polarographic and voltammetric characteristios" of 4-aminopyrimidine and cytosine in DMSO (0.1 M TEAP)

\begin{tabular}{|c|c|c|c|c|c|c|c|c|}
\hline \multirow{2}{*}{$\begin{array}{l}\text { Com- } \\
\text { pound }\end{array}$} & \multirow[t]{2}{*}{$-E_{1 / 2} / \mathrm{V}$} & \multirow[t]{2}{*}{$\left(E_{1 / 4}-E_{3 / 4}\right) / m v$} & \multirow{2}{*}{$\begin{array}{c}I_{\mathbf{d}}^{b} \\
.\end{array}$} & \multirow[t]{2}{*}{$x^{r}$} & \multicolumn{3}{|c|}{$-E_{p} \mu^{d}$} & \multirow{2}{*}{$\begin{array}{l}\text { CPE } \\
n\end{array}$} \\
\hline & & & & & Ic & IYa & IVc & \\
\hline 2-HP' & 1.67 & 340 & 0.77 & $0.5 I$ & $\begin{array}{c}1.80 \\
(228)\end{array}$ & $\begin{array}{c}0.17 \\
(1.32)\end{array}$ & $\begin{array}{c}022 \\
(134)\end{array}$ & 0.58 \\
\hline 4 AP & 263 & 79 & 1.15 & 0.42 & $\begin{array}{c}268 \\
(6.04)\end{array}$ & $\begin{array}{c}0.59 \\
(0.40)\end{array}$ & $\begin{array}{c}0.65 \\
(0.38)\end{array}$ & 1.15 \\
\hline $\begin{array}{c}\text { Cyro- } \\
\text { sine }\end{array}$ & 237 & 117 & 0.81 & 0.50 & $\begin{array}{c}245 \\
(285)\end{array}$ & $\begin{array}{c}0.44 \\
(1.30)\end{array}$ & $\begin{array}{c}0.52 \\
(204)\end{array}$ & 1.04 \\
\hline
\end{tabular}

- Some of the values may be concentration-dependent: daca are at the $2 \mathrm{~m}$ il level.

- Dirrusion current constant: $I_{d}=\bar{i}_{d} / \mathrm{cm}^{2 / 3} t^{1 / 6}$.

- $\log i=X \log h_{\mathrm{H}_{3}}$.

- Numbers in parencheses are the corresponiding cyclic voltammetric curreat functions, $i_{p} / A c^{2 / 2}$, based on $A=26 \mathrm{~mm}^{2}$ for cytosine and $0.50 \mathrm{~mm}^{2}$ for 4AP. the values are 100 times as large for $A$ in $\mathrm{cm}^{2}$.

- Controlled electrode potential electrolysis: $n$ obvinod by onelowest.

Fata from rel 13 are inchuded as a basis for comparison. 


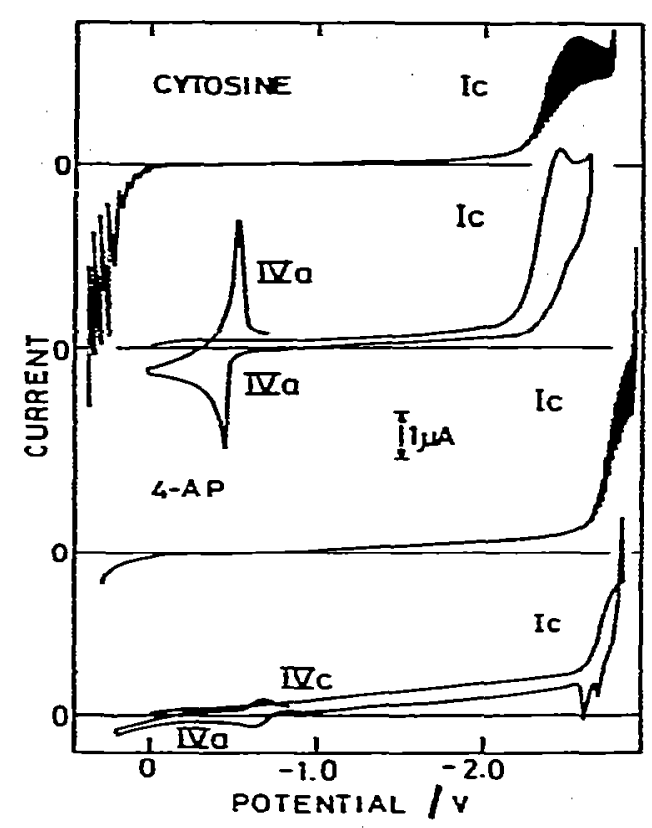

Fig 2 DME polarograms and cyclic volvammograms of $2 \mathrm{~m} \mathrm{Mf}$ cytosine and of $2 \mathrm{~m} \$ 4$-aminopyrimidine (4-AP) in DMSO (0.1 M TEAP). Scan rate in cyclic voltammetry = $69 \mathrm{mV} / \mathrm{s}$ : working electrodes: HMDE (2.6 $\left.\mathrm{mm}^{2}\right)$ for cytosine and MPPDE $\left(0.50 \mathrm{~mm}^{2}\right)$ for 4AP. Roman numbers: polarographic waves and voltammetric peaks involved.

adjustment for solvent viscosity-roughly coincide with those expected from $I_{d}$ for pyrimidine in AN [25], and $I_{d}$ and $i_{p} / A c v^{1 / 2}$ for 2-HP in water [26]. which involve $I$ $e$ processes; however, the values for cytosine are lower. This suggests that electrode process Ic involves (a) a 1 e reaction to produce a free radical followed by its irreversible consumption, e-g- dimerization, for 4-AP and (b) a 1 e reaction followed or accompanied by a parent compound-consuming reaction for cytosine: the nature of the latter reaction is subsequently discussed.

It is also evident that (a) wave and peak Ic for each compound represents reduction of neutral $\mathrm{RH}$, (b) peak IVa, which appears only on the return cycle after passing peak Ic, corresponds to an oxidation involving a product or products produced by the peak Ic process, and (c) peak IVc is due to reduction of the peak IVa process product. As subsequently discussed, the Ic process may produce neutral and/or anionic free radicals, which dimerize, and an anionic form of $\mathrm{KH}$; the peak IVa-IVc redox couple involves the latter anion, eg peaks IVa and IVc grow on repetitive scanning as expected for generation of anion in the peak Ic process. Peak IVa and wave IVa seen on strong base addition are due to the same process.

The appearance of multiple anodic spikes on 4-AP cyclic voltanmograms seems

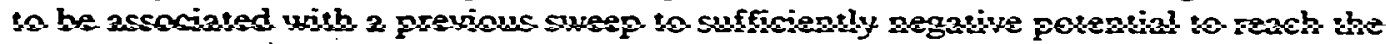
background decomposition region. 
An $i_{d}-c$ plot for cytosine wave Ic is linear up to $5 \mathrm{mM}$; an $i_{\mathrm{p}}-c$ plot is linear up $103.5 \mathrm{~m} M$. Plots of $i_{\mathrm{p}}-\mathrm{o}^{1 / 2}$ are linear at $0.67 \mathrm{~m} M$ cytosine but deviate slightly at 2.4 $\mathrm{m} M$ (o up to $160 \mathrm{~V} / \mathrm{s}$ ). Above $2 \mathrm{~m} M$ cytosine, peak IVa at ca $-0.46 \mathrm{~V}$ starts to grow less steeply with concentation and peak $\Gamma \mathrm{V}(\mathrm{OH})$ appears at ca. $-0.32 \mathrm{~V}$ and grows linearly with concentration.

The shift of $E_{1 / 2}$ for cytosine wave Ic to more positive potential with increasing concentration and nonappearance of a complementary peak la even at 0 of $360 \mathrm{~V} / \mathrm{s}$ further support the notion that the initial 1 e transfer is followed by a rapid irreversible chemical reaction.

The behavior of 4AP in respect to concentration is generally similar to that of cytosine.

\section{Effect of waper addition}

No effect is noticed when the water concentration in DMSO solutions of 4AP or cytosine is $1 \%$ or less. This phenomenon, which differs from. the effect of $\mathrm{H}_{2} \mathrm{O}$ addition on pyrimidine [25] and the azabenzenes [27] in AN, would seem to be due to $\mathrm{RH}$ in each case functioning as a more effective proton donor than $\mathrm{H}_{2} \mathrm{O}$ at low water concentrations in DMSO. Above $1 \% \mathrm{H}_{2} \mathrm{O}$ for $4 \mathrm{AP}$ and $10 \% \mathrm{H}_{2} \mathrm{O}$ for cytosine a new cathodic wave appears at more negative potential than wave Ic; this new wave, whose current markedly increases and whose potential becomes more positive with increasing $\mathrm{H}_{2} \mathrm{O}$ concentration, may correspond to a hydrogen ion reduction, reflecting an incteasing proton activity.

With increasing $\mathrm{H}_{2} \mathrm{O}$ concentration from $1 \%$ to $30 \%$, the wave seen at $-26 \mathrm{~V}$ for 4-AP becomes more positive and markedly increases in magnitude; the wave likely represents a fusion of the $4 \mathrm{AP}$ wave Ic and the wave due to water.

The pattern seen for cytosine is more complicated. Peak and wave Ic shift to more fositive potential and decrease in height above $10 \% \mathrm{H}_{2} \mathrm{O}$ and vanish at $\mathrm{ca} 35 \%$ $\mathrm{H}_{2} \mathrm{O}$. The latter effect is parallelled by the failure of cytosine to exhibit a reduction prve in aqueous media except between pH 3 and 6 [1].

It is evident from these results that the effect of the minute amount of water (less than $0.1 \%$ by volume) added with $\mathrm{HClO}_{4}$ or $\mathrm{Et}_{4} \mathrm{NOH}$ is negligible (cf. subsequent sections).

\section{Effect of strong base addition}

Addition of $\mathrm{Et}_{4} \mathrm{NOH}$ to DMSO (0.1 M TEAP) produces an anodic DME wave at $-0.09 \mathrm{~V}$, whose height increases linearly with $\mathrm{Et}_{2} \mathrm{NOH}$ concentration; above ca. 1 $\mathrm{m} M$, a second anodic wave appears at ca. $-0.6 \mathrm{~V}$, whose height also increases with $E_{1}$ NOH concentration but to a lesser exient On cyclic voltammetry, an apparently complementary cathodic peak also appears at ca $-0.2 \mathrm{~V}$. These waves and peaks 
result from oxidation of mercury in the presence of an anion (hydroxide), which forms an insoluble compound with $\mathrm{Hg}(\mathrm{I})$.

With increasing $\mathrm{Et}_{4} \mathrm{NOH}$ concentration (Fig. 3), wave and peak Ic-corresponding to reduction of $\mathrm{RH}$-decrease and then disappear at $\mathrm{Et} / \mathrm{NOH} / \mathrm{pyrimidine}$ concentration ratio of 1.0 for cytosine and 1.5 for 4-AP. On the other hand peaks $\mathrm{IVa}$ and IVc increase with increasing $\mathrm{Et}_{4} \mathrm{NOH}$ concentration and appear even if the cathodic scan-before reversal-does not reach the potential at which peak Ic appears. Waves IVa(OH) and VIIa in Fig. 3 correspond to the two waves seen on $\mathrm{Et}_{4} \mathrm{NOH}$ addition in absence of $\mathrm{RH}$.

The foregoing and subsequent evidence support peak IVa being due to an oxidation involving the dissociated form (anion) of $\mathrm{RH}$ and peak IVc being due to reduction of the peak IVa product. which is probably a complex or insoluble product of $\mathrm{Hg}(\mathrm{I})$ and pyrimidine anion, $\mathrm{R}^{-}$.

\section{Effect of strong acid addition}

The effect of protonation was examined by adding $\mathrm{HClO}_{4}$ to solutions of cytosine and 4-AP in DMSO up to a ratio of 1.5 acid to 1.0 base (Figs. 4 and 5). Addition of $\mathrm{HClO}_{4}$ alone to DMSO (0.1 M TEAP) produces a DME wave (VIc) at $+1.03 \mathrm{~V}$ $\left(E_{\mathrm{p}}=-1.16 \mathrm{~V}\right)$, whose height is linearly proportional to $\mathrm{HClO}_{4}$ concentration.

On $\mathrm{HClO}_{4}$ addition to a DMSO solution of $\mathrm{RH}$, a new wave (IIc) appears at more positive potential than original wave Ic and grows at the expense of wave Ic. which has completely disappeared by an acid-pyrimidine ratio of 1.0 for 2-HP [13] and 1.5 for 4-AP and cytosine. Wave VIc (due to reduction of $\mathrm{H}^{+}$derived from $\mathrm{HClO}_{4}$ ) appears at an acid-pyrimidine ratio of about 1.0 for all three compounds. It is

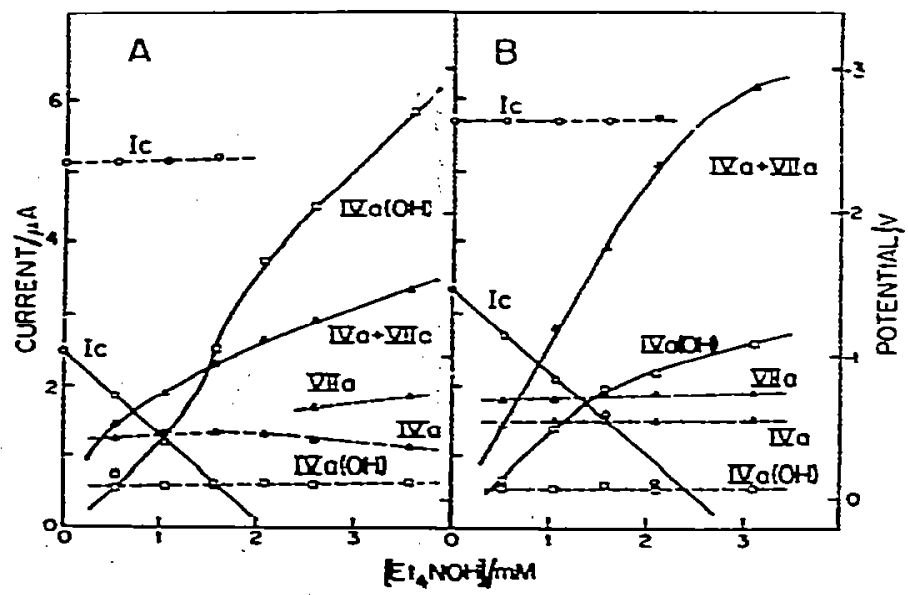

Fig 3. Effect of addition of a strong base (E1,NOH) on the DME polarographic behavior of (A) Cy iosine ( $2 \mathrm{~mA})$ and (B) 4 -aminopyrimidine $(2 \mathrm{mM})$ in DMSO (0.1 M TEAP). ( - - ) Potential: ( $-\longrightarrow$ current Roman numbers: polarographic waves involved. 


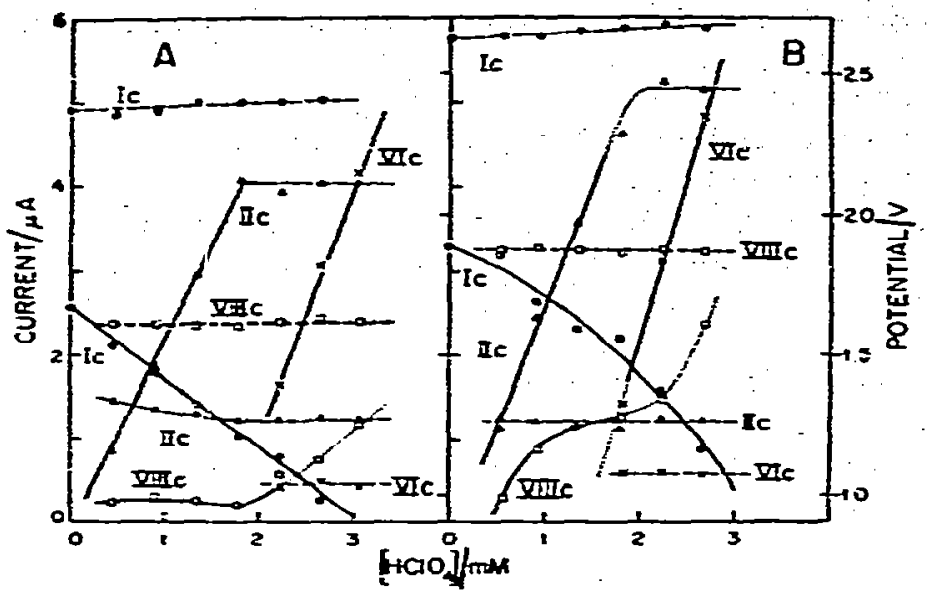

Fiz 4. Eltect of addition of a strong acid (perchloric acid) on the DME polarographic behavior of (A) cytosine (2 m/f) and (B) 4-aminopyrimidine (2 mM) in DMSO (0.1 M TEAP). (- - -) Potential: (-) current. Roman numbers: polarographic arves involved.

difficult to measure the heights of the two waves at higher acid/base ratios because of the appearance of maxima and drop-time irregularities when the acid concentration reaches $3 \mathrm{mM}$. There is also the situation that the $\mathrm{H}^{+}$concentration at the electrode surface is less than that of the bulk solution due to the $\mathrm{H}^{+}$consuming reduction producing peak VIIIc. As a result, the $\mathrm{RH}$ concentration at the electrode

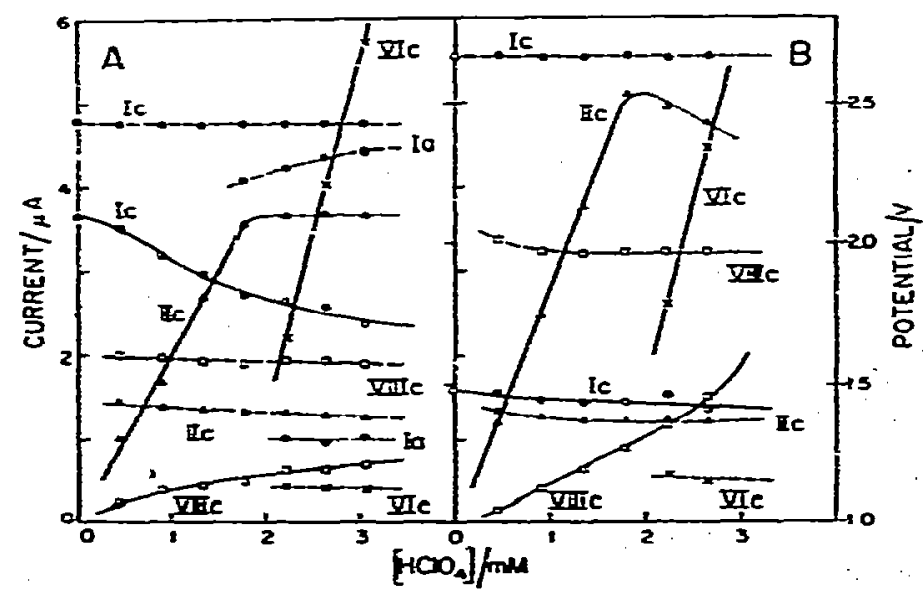

Fig 5. Effect of addition of a stroog acid (perchloric acid) on the cydic voltanmetric betavior of (A) cytosine ( $\left.2 \mathrm{~m} M ; A=26 \mathrm{~mm}^{2}\right)$ and $(B) 4$-aminopyrimidine $\left(2 \mathrm{mM} ;=0.50 \mathrm{~mm}^{2}\right.$ ) in DMSO (0.: $M$ TEAP) ( - - ) Potential; (——) current. Roman mumbers: voliammetric peaks invohed. 
surface exceeds that in the bulk solution due to the equilibrium shift,

$\mathrm{RH}_{2}^{+} \rightleftharpoons \mathbf{R H}+\mathbf{H}^{+}$

A solution containing cytosine and $\mathrm{HClO}_{4}$ in $1.0: 0.9$ ratio (Fig. 6) shows waves of $E_{t / 2}=-1.27$ and $-2.40 \mathrm{~V}$ (relative height ratio, corrected for drop-time, is $3.4: i)$, due to reduction of protonated and unprotonated cytosine, respectively. A cyclic voltammogram shows four cathodic peaks and a minute anodic peak (Fig. 6). On increasing $v$, all of the currents increase but in markedly different fashion. The Ic-Ia peak pair probably represents the cytosine-radical anion redox couple peak IIc reduction of protonated cytosine, peak VIIIc reduction of adsorbed protonated cytosine and protonated free radical, and the peak at $-21 \mathrm{~V}$ reduction of an impurity as previously mentioned. The variation in relative peak heights $\left(i_{\mathrm{p}} / c v^{1 / 2}\right.$ values) with scan rate $\mathfrak{v}$, (Table 2 ) is explicable on the basis, for example, that, as 0 increases. less protonated cytosine (species producing wave IIc) is formed from unprotonated cytosine (wave Ic species) reacting with other protonated species (wave IIIc source) due to equilibrium shifts as protonated cytosine is reduced. This is supported by the approximate constancies with $v$ of the sum of the current functions for peaks IIc and VIIIc $(7.8 ; 8.6 ; 8.3)$, i.e, sum of protonated species. and of the sum of peaks Ic, IIc and VIIIc $(13.0 ; 13.3 ; 12.2)$, i.e, sum of RH and protonated species. The summation of peaks Ic and VIIIc is also constant at the two higher scan rates: $6.2 ; 10.4 ; 10.6$.

The variations in the polarographic and voltammetric patterns are consistent with a prior protonation of the pyrimidine, probably at $N(3)$, to give a species more easily reducible than the original molecule [25]. The fact that the 2-HP wave disappears at a $1: 1 \mathrm{acid} /$ pyrimidine ratio [13] but that those of 4-AP and cytosine do not. suggests that prior protonation of cytosine and 4-AP requires a greater hydrogen ion activity than for 2-HP; this is in agreement with their respective aqueous $\mathrm{p} K_{2}$ values.

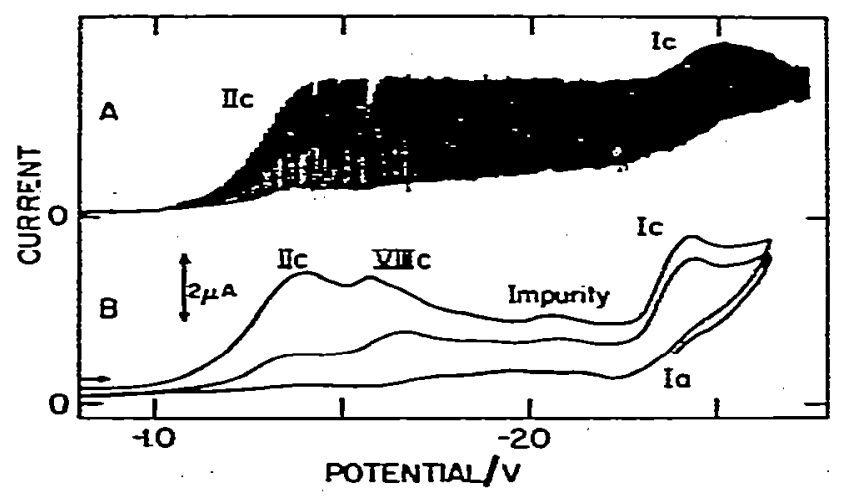

Fig 6: DKE polarogram (A) and cyclic voltammogram (B) for a DMSO (0.1 M TEAP) solution. $20 \mathrm{~m} \mathrm{M}$ in cytosine and $1.8 \mathrm{mM}$ in HCO,. Roman numbers: polarographic waves and voltammetric peaks involved. 
TABLE 2

Cyctic roltammetry of protonated cyrosine in DMSO".

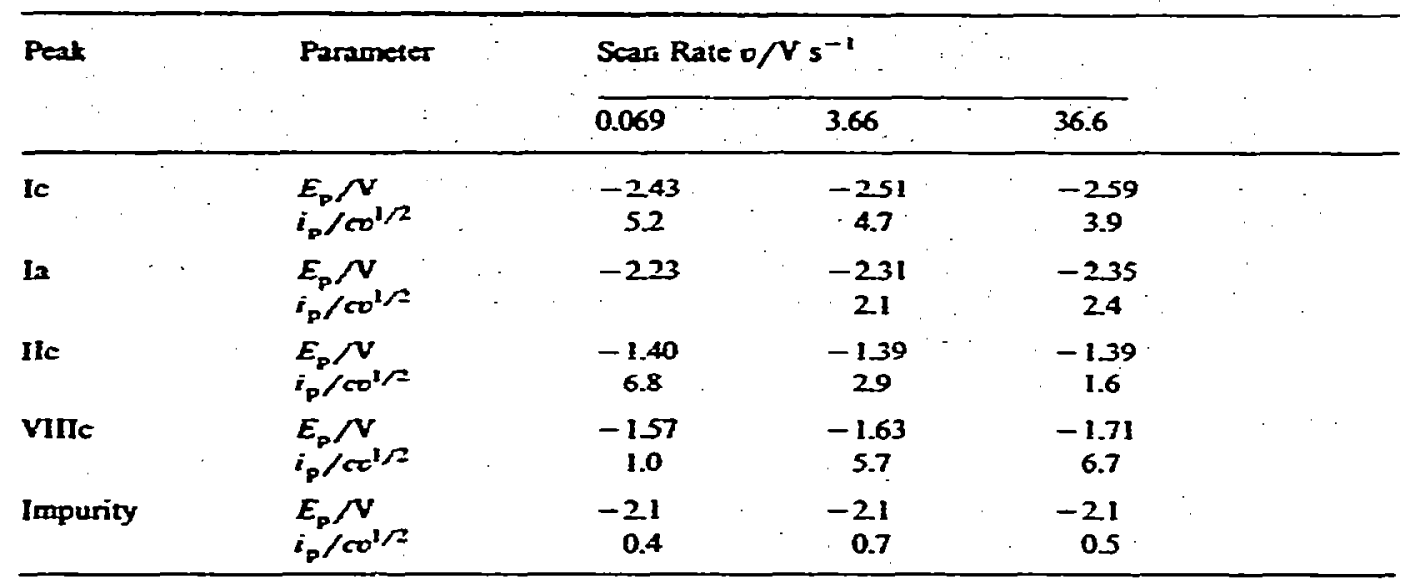

- Solution composition: Q1 M TEAP, $20 \mathrm{mM}$ cytosine. $1.8 \mathrm{mM} \mathrm{HCIO}_{3}$

There is the alternative but related explanation of the need for a higher $\mathrm{HClO}_{2}$ base ratio for disappearance of wave ic for 4-AP and cytosine than for 2-HP, in the fact that protonated 2-HP is reduced at less negative potential than $\mathrm{HClO}_{4}$ itself whereas protonated 4-AP and cytosine are reduced at more negative potential. Consequently, dissociation of the latter species as the free $\mathrm{HClO}_{4}$ is reduced, may be relevant.

Peak pair IVa and IVc at -0.5 to $-0.6 \mathrm{~V}$ (Table 1), which involves mercury and an anionic form of each base disappears completely on addition of a small amount of strong acid.

\section{Effect of weak acid addirion}

Addition of a weak acid itself to DMSO (0.1 $M$ TEAP) produces a bydrogen ion reduction wave (VIc) at $+1.8 \mathrm{~V}$ (chloroacetic acid) or $-21 \mathrm{~V}$ (benzoic acid), whose height is linearty proportional to concentration. As might be expected from chloroacetic acid being somewhat more highly dissociated than benzoic the increase in current with concentration is somewhat greater for chloroacetic; $E_{1 / 2}$ in benzoic acid solution shifts positively with increasing concentration.

As either acid is added to a solution of 4-AP or cytosine in DMSO up to an acid/base ratio of 2 (Fig 7), a new wave (III) appears at a potential corresponding to the acid strength and grows as wave Ic decreases; similar to the behavior on $\mathrm{HClO}_{4}$ addition; however, the wave patterns are quite complicated, reflecting the weaker protonating ability of these acids as compared to $\mathrm{HCOO}_{4}$ and the resulting equilibria involved, $c, g$, formation of adducts between nitrogen base and undissoci- 


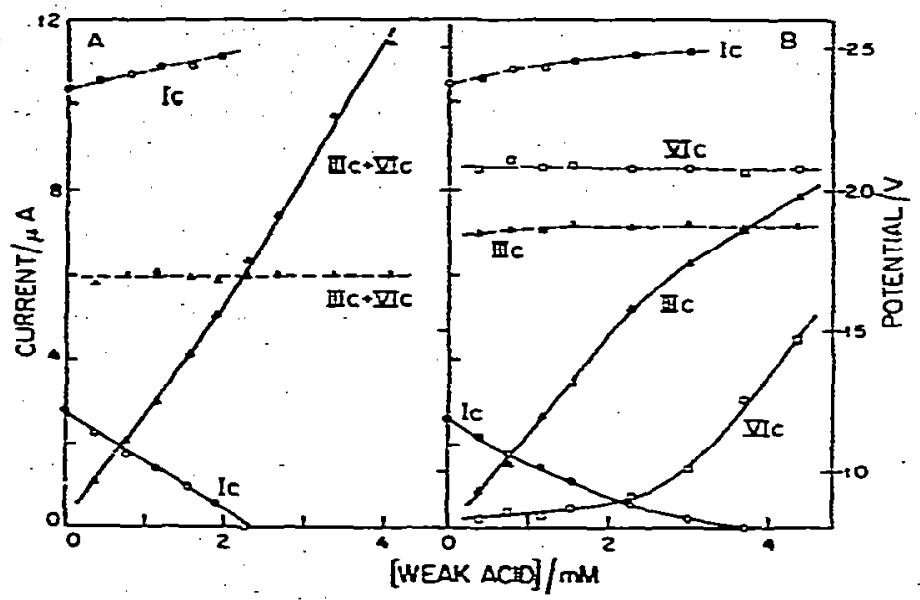

Fig 7. Effect of addition of weak acid (A: chlorozcetic acid; B: benzoic acid) on the dme poiarographic behavior of cytosine $(2 \mathrm{m.M})$ in DMSO (0.1 M TEAP). (- $(-)$ Potential; (- - -) current. Roman numbers: polarographic waves involved.

ated acid. Further complication results from possible reaction of reduction products with acid to produce further reducible species.

On chloroacetic acid addition, the original cytosine wave Ic vanishes by an acid/base ratio slightly exceeding one with the one new wave involving a combination of cytosine-acid adduct and acid reduction. The 4-AP wave decreases to one-third its original magnitude at an acid/base ratio of 2; the new wave also seems to be a combined wave.

On benzoic acid addition, cytosine wave Ic has disappeared by an acid/base ratio of 2; a new wave-more positive than the benzoic acid wave-appears and grows, while a small benzoic acid wave starts to grow in magnitude as the acid/base ratio exceeds one. The 4-AP wave linearly decreases to zero at an acid/base ratio of 2 , while a wave at the benzoic acid reduction potential steadily increases; the latter wave is also a combined wave arising from reduction of both base-acid adduct and acid itself.

Cyclic voltammograms show generally similar behavior with some variation arising from the difference in time scale and the electroactivity due to reaction products, eg-x a redox couple $(\mathrm{Va}, \mathrm{c})$, due to a $\mathrm{Hg}(\mathrm{I})-\mathrm{Hg}(\mathrm{O})$ couple involving the acid anion, appears at about $-0.1 \mathrm{~V}$ in the case of benzoic acid and $+0.2 \mathrm{~V}$ in the case of chloroacetic acid.

\section{Controlled potential electrolysis and coulometry. Compasition of electrolysis products}

Cytosine was electrolyzed at $-2.6 \mathrm{~V}$ and 4-AP at $-2.7 \mathrm{~V}$; coulometric $n$ data are summarized in Table 1. Typical polarograms obtained during the course of electroly- 
sis are shown in Figs 8 and 9; the variation of current with time during electrolysis is shown in Fig 10. Certain characteristics of these figures are immediately apparent.

Each $\log i-t$ curve indicates involvement of at least two electrolytic processes with a relatively slow intervening chemical step: The first process, which predominates early in the electrolysis, is strongly dependent on RH concentration and probably involves the father-son reaction. As the RH concentration decreases, the effect of follow-up chemical reactions on the effective rate of electrolysis decreases.

During electrolysis, anodic wave IVa involving the electrolytic reduction product of each pyrimidine appears and grows at the expense of original $\mathrm{RH}$ reduction wave Ic; the sum of waves Ic and IVa for cyrosine is nearly constant but that for 4-AP increases somewhat. $E_{1 / 2}$ values of the anodic wave $(-0.6 \mathrm{~V}$ for $4-\mathrm{AP} ;-0.4 \mathrm{~V}$ for cytosine) essentially coincide with those for the oxidation waves obtained on strong base addition (Fig - 3).

After electrolysis was complete increments of deoxygenated perchloric acid were added to the electrolyzed solution; the variations in the polarographic patterns resulting from such addition are summarized in Figs. 11 and 12 . On continued addition of $\mathrm{HClO}_{4}$ to the electrolyzed solutions (Figs. 8 and 9), original wave Ic grows at the expense of wave IVa and, then, at $\mathrm{HClO}_{4}$ to original $\mathrm{RH}$ ratio of about I. $5: 2$, wave IVa disappears, wave IIc-corresponding to reduction of protonated RH-appears and grows at the expense of wave Ic, and wave VIIIc due to reduction of protonated radical produced by decomposition of dimer in presence of excess

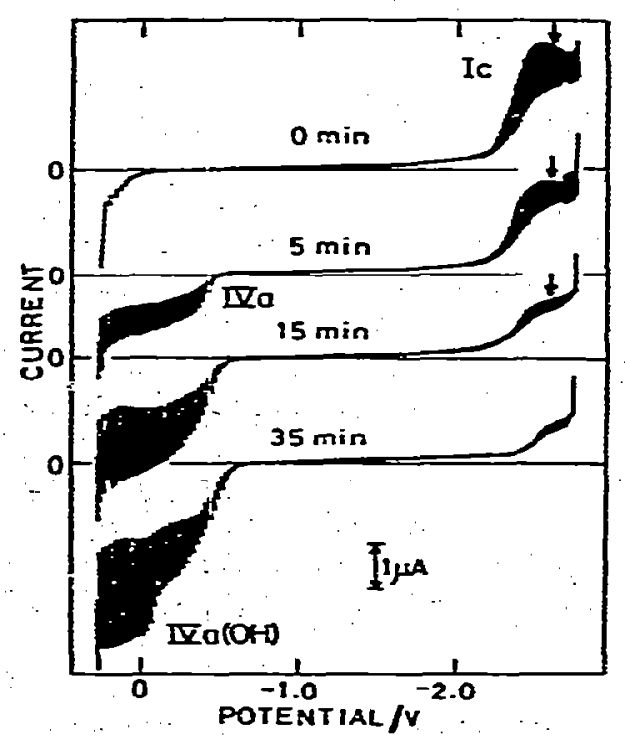

Frg 8. Polarograms of cytosine (1.73 m M) solntion during its controlled electrode potential electrolysis at $-26 \mathrm{~V}$ in DMSO (0.1 M TEAP). Time in minutes after start of electrolysis is noted on each curve Arrowheads indicate the electrof ssis potential ( $-26 \mathrm{~V})$ 
hydrogen ion due to strong acid appears and grows. The ratio of the maximum heights of wave IVa; regenerated wave Ic protonated $R H$ wave IIc and wave VIIIc compared to origival wave Ic before electrolysis are approximately $1.1,0.6 .0 .9$ and 0.6 , respectively, for cytosine, and $1.2,0.8,1.3$ and 0.6 , respectively, for 4-AP.

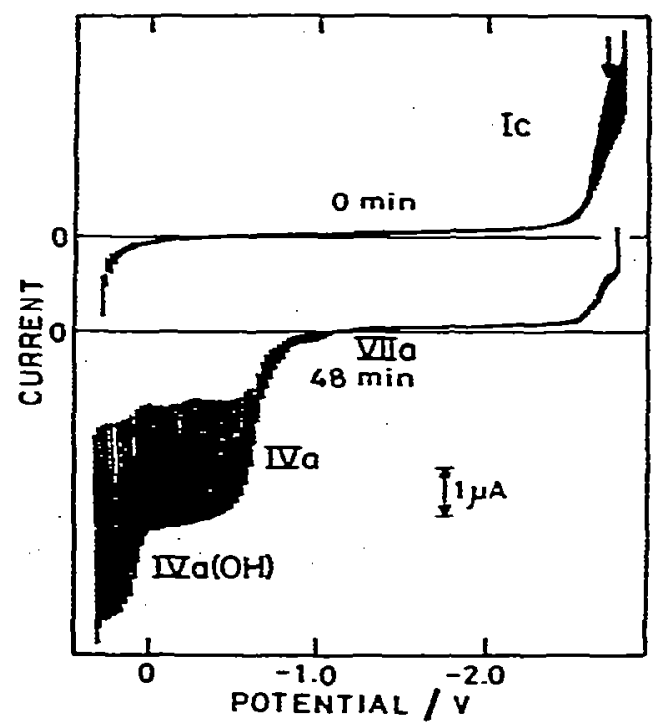

Fig 9. Polarograms of 4-aminopyrimidine $(2 \mathrm{~m} \boldsymbol{M})$ solution during its controlled electrode potential electrolysis at $-27 \mathrm{~V}$ in DMSO (0.1 M TEAP). Time in minutes after star of electrolysis is noted on each curve Arrowhead indicates the electrolysis potential $(-27 \mathrm{~V})$.

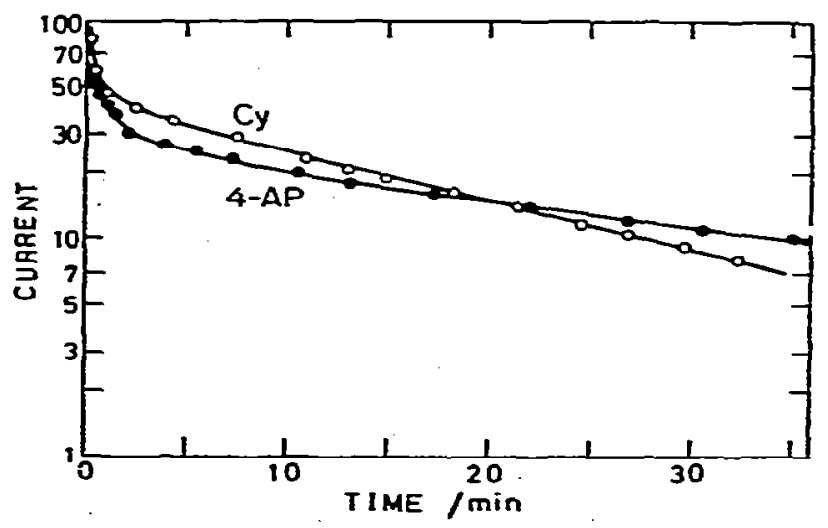

Fig. 10. Variation of the current (in arbitsry units) with time during the controlled electrode potential clectrolysis of $2 \mathrm{mM} 4$-aminopyrimidine (4-AP; $\ominus$ ) and of $1.73 \mathrm{mM}$ cytosine (Cy: O) in DMSO (O.1 M TEAP). 
The variations in wave pattern during electrolysis and on subsequent $\mathrm{HClO}_{4}$ addition are elucidated in the folloving section on Reaction Mechanisms.

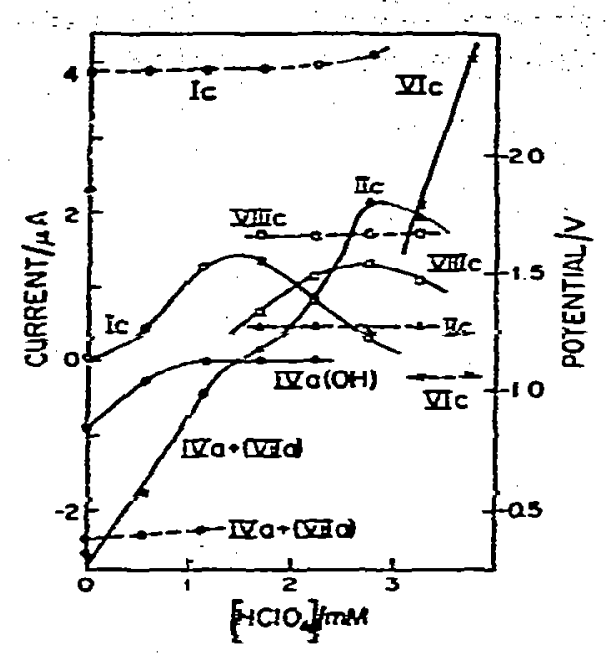

Fig. I1. ETlect of $\mathrm{HClO}_{4}$ addition on an exhaustively electrolyzed solution of 1.73 m. 4 cytasine in DMSO (0.0 $M$ TEAP]. Positive currents are gibodic, negalive currents are anodic The double circies at $0 \mathrm{~m} M$ HClO, show the current and potential of the cytosine arve seen in the unelectrolyzed solution. (- - - ) Potentivi; (- $)$ carrent. Roman numbers: polarographic waves involved.

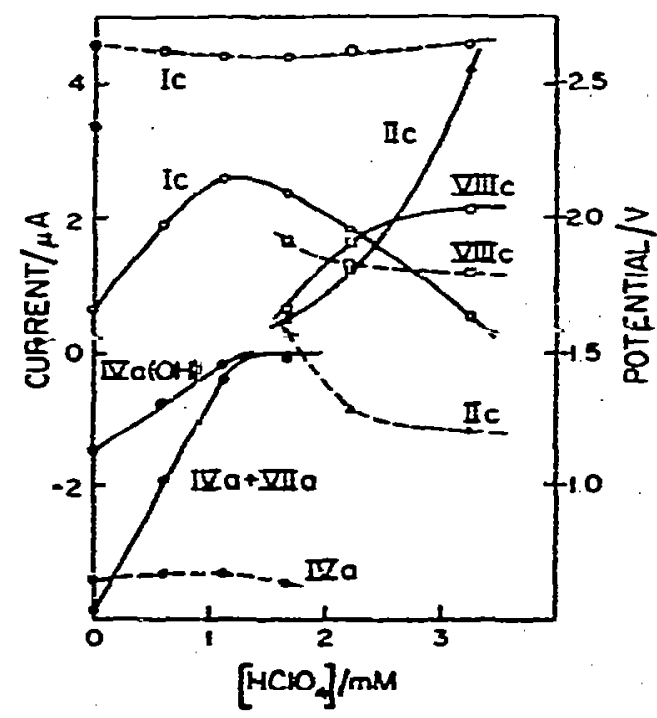

Fi2 12 Effect of HCO, addition an an exhustively electrolyed solution of $2 \mathrm{~m} M$ 4aminopyrimidine in DMSO (0.1 M TEAP2. Pocitive curreats are cabodic; negative currents are apodic. The double circles al 0 mM HCO, show the current and pocential of the 4AP wave seen in the unelectrolyzed solution (- - Petentivl; ( ) current Romen numbers: polirographic waves involved. 


\section{REACTION MECHANISMS}

The electrolytic redox reaction paths in DMSO for 4-aminopyrimidine (4-AP) and cytosine, based on the avalable evidence and including accompanying chemical reactions, are summarized in Fig. 13, together with an assignment of the waves and peaks, and of the approximate potentials associated with each wave and peak: the latter potentials are also tabulate 1 in Table 3 , to which the data on pyrimidine and 2-hydroxypyrimidine (2-HP) in nonaqueous media have been added for comparison. In the subsequent discussion, $\mathrm{RH}$ is used to designate a pyrimidine molecule with a removable, i.e, possibly acidic, proton.

In DMSO (0.1 M TEAP), in the absence of added hydrogen or hydroxide ion source, each pycimidine undergoes an apparently reversible 1 e reduction (wave or peak Ic) to form a radical anion, which is a strong base and which can dimerize to a diau on species. Dimerization, which involves reaction of similarly charged species. seem. less rapid than the attack of the radical anion on unreduced pyrimidine to abstract a proton, producing the neutral free radical, which dimerizes much more rapidly than the corresponding radical anion, and the anion of the pyrimidine. The latter anion is involved in a $\mathrm{Hg}(\mathrm{I})-\mathrm{Hg}(\mathrm{O})$ couple ( $\mathrm{Va}-\mathrm{IVc}$ ); the sum of the current magnitudes for waves Ic and IVa should approximate that expected for a $1 e$

(o)

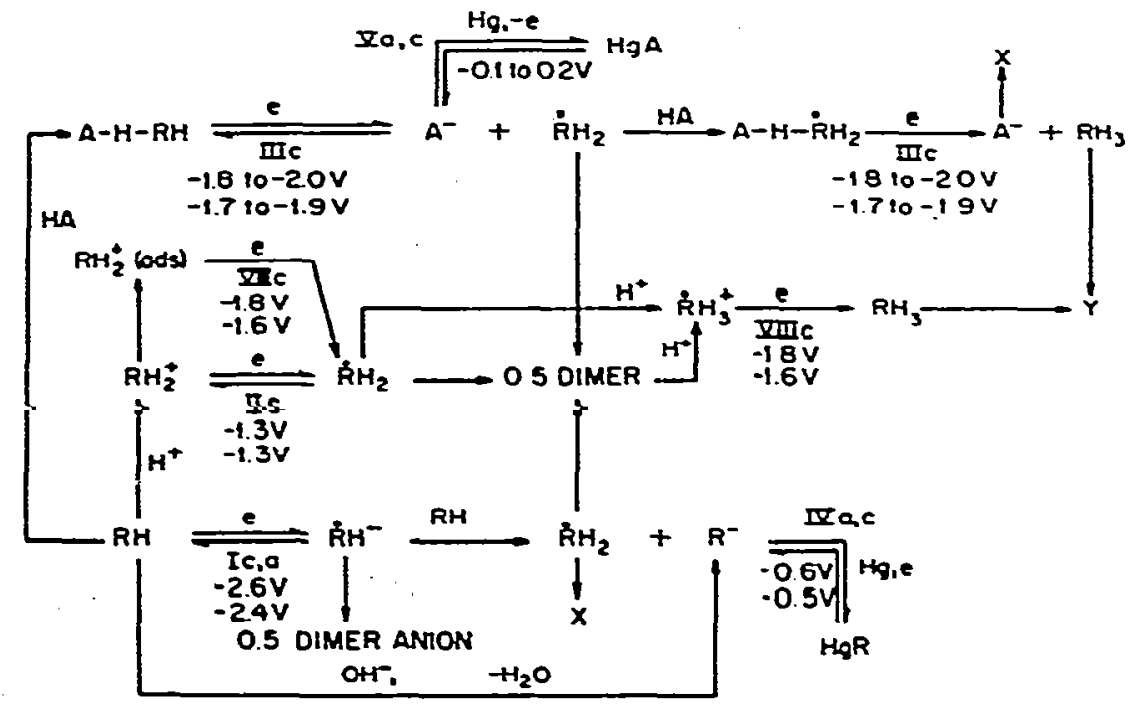

Fig. 13. Interpretation of the electrochemical and related chemical behavior observed for 4-aminopyrimidine and cytosine (RH) in DMSO (O.1 M TEAP) solution in the presence of (a) weak scid (HA), (b) strong acid ( $\left.\mathrm{H}^{+}\right)$(c) no added acid or base, and (d) strang base (BOH). All of the acid-base reactions shown are presumably reversible. Where two sets of potentials are given. the upper refers to 4 aminopyrimidine and the fower to cytasine $X$ indicates that subsequent reactions for the species involved are shown elsewhere on the figure. $Y$ refers to the deamination reaction which is followed by reduction of the resulting product 
TABEE 3

Potentialse at which waves and peaks seen in pyrimidine redox patterms in DMSO occur

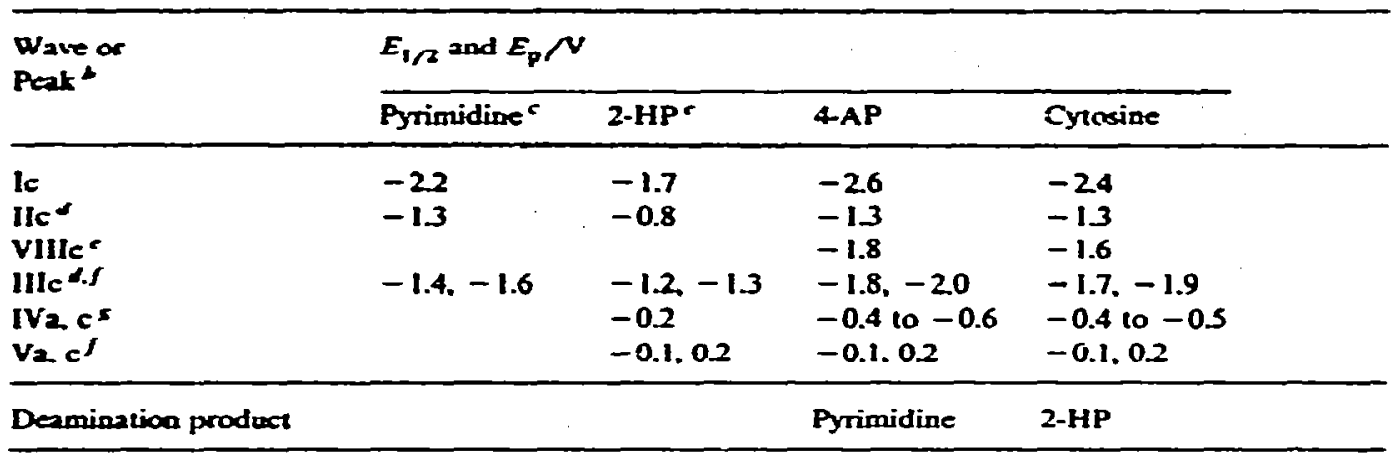

- Potentials are referred to the aqueous SCE

- Average wave and peat patterns are schematically indieated in Fig 13.

- Data raken from refs. 13,28 and 29.

- Wave or peak Vle due to hydrogen ion reduction oceurs at $-1.1 \mathrm{~V}$ for $\mathrm{HClO}_{4}$. $-1.8 \mathrm{~V}$ for chloroacetic acid and $-21 \mathrm{~V}$ for bemoic acid.

The process producing the weve or peak may involve addition of a second electron to the pyrimidine.

The firs value is for chloroactic acid solution.

- A similar wave or peak pair appears in presence of hydroxide anion at - C.I V (wave IVa(OH)): as higher hyoiraxide concentration, a second pair appears at $-0.6 \mathrm{~V}$ (wave VIIa).

faradaic process except that the nature of the IVa-IVc couple, which may involve a film, and the very negative potential needed for 4-AP reduction, e.g., possible solvent and water reduction to generate $\mathrm{OH}^{-}$ions, introduce complications.

A possible concurrent route, which does not alter the principal arguments involved, is that of the proton exchange involving in addition to reaction between RH and radical anion $\dot{R H}^{-}$, reaction between $\mathrm{RH}$ and the anionic dimeric species formed by $\mathrm{RH}^{-}$. Favoring the latter reaction is the likelihood of the dimer anion being a stronger base than the radical anion itself. Favoring the anion radical as the proton acceptor is the probably more favorable kinetics for reaction between $\mathrm{RH}^{-}$ and $\mathrm{RH}$ as compared to that between $\mathrm{RH}^{-}$and $\dot{\mathrm{RH}}^{-}$.

The electrochemical reduction site, based on prevously observed reductions of pyrimidines in aqueous and nonaqueous media $[1,25,30,31]$ is the $3,4 \mathrm{~N}=\mathrm{C}$ double bond. Dimesization probably involves formation of a $4,4^{\prime}$ or $6,6^{\prime} \mathrm{C}-\mathrm{C}$ bond. The anionic species formed can neutralize their charge by ion-pairing with cationic Lewis acids such as protons from added acids and acidic impurities or traces of metal ions.

On addition of a strong base (hydroxide ion) to an RH solution, all of the pyrimidine is converted to the anion $\mathbf{R}^{-}$and the only electrochemical activity seen is that due to its couple with mercury (IVa-IVc). The proton removed from RH on its zeaction with a strong base, e.g, its radical anion or hydroxide ion, is probably the proton on $N(1)$ in the case of cytosine and on $C(2)$ in the case of 4-AP.

On addition of a strong acid, i.e, a freely available hydrogen ion source, the 
protonated pyrimidine ( $\mathrm{RH} \cdot \mathrm{H}^{+}$) formed is more easily reduced (wave llc) than the neutral RH (Ic) with the resulting IIc current generally exceeding that for Ic; the couple due to reaction of $\mathrm{R}^{-}$with mercury (IVa-IVc) is not seen. Based on the protonation patterns seen in aqueous media and the increased ease of reduction on protonation, the most likely protonation site in $\mathrm{RH}$ is $\mathrm{N}(3)$. The neutral free radical formed can dimerize or, when the proton/pyrimidine ratio exceeds 1 . can be protonated and reduced to the 3,4-dihydropyrimidine $\left(\mathrm{RH}_{3}\right)$. If the latter is derived from 4-AP or cytosine, $C(4)$ is the site of a gem diamine and deamination can occur to regenerate the $3,4 \mathrm{~N}=\mathrm{C}$ bond, forming pyrimidine itself in the case of 4-AP and 2-HP in the case of cytosine $[1,5,10]$. Since pyrimidine and $2-\mathrm{HP}$ are respectively. more easily reducible than 4-AP or cytosine, they are reduced as they are formed. contributing to the faradaic current.

Addition of a weak acid to. an $\mathrm{RH}$ solution results in formation of an adduct between the acid and $\mathrm{RH}$ as a result of hydrogen bridging between the acid proton and the pyrimidine reduction site, probably at $\mathrm{N}(3)$, since reduction of the pyrimidine in the adduct is facilitated (wave IIIc). The free radical produced can dimerize or can hydrogen-bond another molecule of acid to form an adduct reducible within the available potential range to produce a dihydropyrimidine, which, as noted, can deaminate in the case of 4-AP and cytosine to produce a reducible compound (pyrimidine or 2-HP). The weak acid anion. liberated on reduction of the adduct. can produce a $\mathrm{Hg}(\mathrm{I})-\mathrm{Hg}(0)$ couple similar to the pyrimidine anion; the resulting wave or peak couple $(\mathrm{Va}-\mathrm{Vc})$, however, occurs at a potential characteristic for the acid involved. On the other hand, the mercury-pyrimidine anion wave (IVa-IVc) occurs at a potential characteristic of the pyrimidine.

The causes for the coulometric $n$ values for cytosine and 4-AP (Table 1) being about twice those for 2-HP are probably a combination of (a) decreased effect of the father-son reaction, especially as the $\mathrm{RH}$ concentration decreases during electrolysis, (b) reduction of the neutral free radical, $\dot{\mathrm{R}} \mathrm{H}_{2}$, resulting from protonation by $\mathrm{RH}$ of the initially produced radical anion in the father-son reaction, i.e., an ECE process, (c) reduction of the 2-HP or pyrimidine formed on deamination of the $\mathrm{RH}_{3}$ product of the preceding reduction under the condition of no added proton source. and (d) reduction of the protonated free radical (wave VIIIc process). A possible additional cause for $n$ approaching or exceeding one for cytosine and 4-AP is the reduction of these compounds to the radical anion, which neutralizes its charge by reaction with a solution species, eg., $\mathrm{Na}(\mathrm{I})$ impurity, to form a neutral ion-pair. which can be reduced in a second $l e$ process as well as dimerize.

The data obtained on controlled potential electrolysis (CPE), coulometry, and subsequent treatment of the electrolyzed solution with $\mathrm{HClO}_{4}$ are explicable on the basis of the mechanisms discussed. During CPE which involves the wave Ic process possibly coupled with a father-son reaction,

$\mathbf{R H}+e \rightarrow \dot{\mathbf{R}} \mathbf{H}^{-}$

$\dot{\mathrm{R}} \mathrm{H}^{-}+\mathrm{RH} \rightarrow \dot{\mathrm{R}} \mathrm{H}_{2}+\mathrm{R}^{-}$ 
half to all of the $\mathrm{RH}$ is reduced to a radical species, which, in turn dimerizes, is reduced or is converted to a reducible species; the remainder is converted to the anion (eqn. $T$ which causes wave IVa,

$\mathbf{R}^{-}+\mathbf{H g} \rightarrow \mathbf{H g R}+\mathbf{e}$

The latter wave is similar to the anodic wave produced on adding strong base to an RH solution.

Addition of $\mathrm{HClO}_{4}$ converts $\mathrm{R}^{-}$to $\mathrm{RH}$, thus decreasing wave IVa (eqn. 8) and regenerating RH, which produces wave Ic (eqn. 6); the maximum height of regenerated wave Ic is a fraction (about 61\% for cytosine; $73 \%$ for 4-AP) of that of wave Ic before CPE which height is reached when the added $\mathrm{HClO}_{i}$ concentration equals a similar fraction (about $65 \%$ for cytosine; $76 \%$ for 4AP) of the original RH concentration (Figs. 11 and 12). (It must be kept in mind that original wave Ic represents neduction of one-half to all of the $\mathrm{RH}$ present.) These facts suggest that the CPE involves additional proton and electron consuming reactions such as reduction of $\dot{R} \mathbf{H}_{2}$ and of deamination products of $\mathbf{R H}_{3}$. RH,

Further addition of $\mathrm{HClO}_{4}$ furnishes proton donor for protonation if regenerated

$\mathbf{R}^{-}+\mathbf{H}^{+} \rightarrow \mathbf{R H} \stackrel{\mathbf{H}^{+}}{\rightarrow} \mathbf{R H}_{2}^{+}$

The protonated $\mathrm{RH}$ produces wave IIC,

$\mathrm{RH}_{2}^{+}+e \rightarrow \dot{\mathrm{RH}}_{2}$

Continued $\mathrm{HClO}_{4}$ addition beyond that shown in Figs. 11 and 12 does not seem to affect wave Ilc but results in the appearance and growth of wave VIc due to hydrcgen ion reduction. (Precise measurement of wave IIc height is complicated by the large maximum of wave VIc and drop-time irregularity.)

On $\mathrm{HClO}_{3}$ addition to electrolyzed solutions of cytosine (Fig. 11) and 4-AP (Fig. 12), wave VIIIc ( -1.9 to $-1.8 \mathrm{~V}$ for $4 A$, $;-1.66 \mathrm{~V}$ for cytosine), which appears at an acid/pyrimidine ratio of about $1-1.5 / 2$ seims to correspond to the wave VIIIc and peak VIIIc seen in $\mathrm{HClO}_{4}$-containing solutions of cytosine and 4-AP (Figs. 4 and 5; Table 2). The species producing wave VIIIc is probably the free radical $\dot{R}_{2}$ produced by the reduction (wave IIc) of the protonated cytosine or protonated 4-AP. and/or the decomposition product of their dimers in the presence of excess acid [1].

\section{ACKNOMLEDGEMENTS}

The authors thank the National Science Foundation, which helped support the work described, and the University of Osaka Prefecture for a fellowship given io T.W.

\section{REFERENCES}

1_DL_ Smith and PJ. Elving. J. Am Chem Soen 84 (1962) 2741.

2 E Palocet and B. Janik, Arch Biochem Bioplys; 98 (I962) 527. 
3 B. Janik and E Palecek, Arch. Biochem. Biophys, 105 (1964) 225.

4 G. Dryhurst and P.J. Eving. Talanta, 16 (1969) 855.

5 B. Czochralska and D. Shugar, Experientia Suppl. 18 (1971) 251.

6 J.W. Webb, B. Janik and PJ. Elving. J. Am. Chern. Soc. 95 (1973) 991.

7 J.W. Webb, B. Janik and PJ. Elving. J. Am. Chem Soc 95 (1973) 8495.

8 B. Brezina and J. Volke in G.P. Elis and G.B. Wert (Eds.). Progress in Medicinal Chemistry. Vol. 12. North-Holland, Amsterdam; 1975, pp. 247-92

9 A.L Underwood and R.W. Bumett in A.J. Bard (Ed.). Electrosnalyuical Chemistry. Vol. 6. Marcel Dekker, New York, 1973. Ch. 1.

20 PJ. Elving in G. Mrilazzo (Ed). Topies in Bioclectrochemistry and Bioenergeties. Vol. 1. John Wiley. Landon. 1976, PP. 179-286.

11 G. Dryhurst, Eloctrochemistry of Biological Molecules, Academic Press, New York. 1977.

12 B. Czochralska and PJ. Elving. Electrochim Acta, 26 (1981) 1755.

13 T. Wase and P.J. Elving. J. Electroanal Chem, 91 (1978) 249.

14 T.E Cummings and PJ. Eving. J. Electroanal. Chem. 94 (1978) 123.

15 T.E Cummings and P.J. Eving, J. Electroanal. Chem. 102 (1979) 237.

16 W.T. Bresnahan, T.E Cummings and P.). Elving. Electrochim. Acta, 26 (1981) 691.

17 PJ. Eving. Can. J. Chem. 55 (1977) 3392

18 D.G. Brown. J. Soc Chem. Ind. 69 (1950) 353.

19 I.M. Kolthoff and J.E Coctzee, J. Am. Chern. Soc. 79 (1957) 870.

20 K.S.V. Santhanam and P.J. Eving. J. Am. Chem. Soc. 95 (1973) 5482

21 K. Nakanishi. N. Surzuki and F. Yamazaki. Bull Chem. Soc. Japan. 34 (1961) 53.

22 I. Wempen and J.J. Fox. J. Am. Chem. Soc, 86 (1965) 2764.

23 J.S. Kwiatkowski and B. Pullman in A.R. Katritzky and A.J. Boutton (Eds.), Advances in Heterocyelic Chemistry, Vol. 18. Academic Press, New York, 1975, Pp. 199-335.

24 T. Malinski. W.T. Bresnahan. T.E Cummings and P.J. Elving. Bull Soc. Chim. FT. (1980) 410.

25 J.E O'Reilly and P.J. Elving. J. Am. Chem. Soc, 93 (1971) 1871 .

26 T. Wass and P.J. Eling, unpublished rork

27 J.E O'Reilly and P.J. Eving. J. Am. Chem. Soc. 94 (1972) T941.

28 J.E O'Reilly and P.J. Eving. J. Am. Chem Soc. $\$ 3$ (1971) 1871.

29 J.E O'Reilly and P.J. Elving. J. Am. Cheril Soc, 94 (1972) 7941.

30 B. Czochralska and D. Sugar. Biochim. Biophys. Acta. 281 (1972) 1.

31 PJ. Eving, SJ. Pace and J.E O'Reilly, J. Am. Chem. Soc, 95 (1973) 647. 